\title{
Analysis of core genes for colorectal cancer prognosis based on immune and stromal scores
}

\author{
Yi Zhu ${ }^{\text {Corresp., } 1}{ }^{1}$, Yuan Zhou ${ }^{1}$, HongGang Jiang ${ }^{1}$, ZhiHeng Chen ${ }^{1}$, BoHao Lu ${ }^{1}$ \\ ${ }^{1}$ Department of Gastrointestinal Surgery, The Affiliated Hospital of Jiaxing University, Jiaxing, China \\ Corresponding Author: Yi Zhu \\ Email address: zhuanwen456@163.com
}

Background. Colorectal cancer (CRC) is one of the most common malignancies. An early diagnosis and an accurate prognosis are major focuses of CRC research. Tumor microenvironment cells and the extent of infiltrating immune and stromal cells contribute significantly to the tumor prognosis. Methods. Immune and stromal scores were calculated based on the ESTIMATE algorithm using the sample expression profile of the TCGA database. GSE102479 was used as the validation database. Differentially expressed genes whose expression was significantly associated with the prognosis of CRC patients were identified based on the immune matrix score. Survival analysis was conducted on the union of the differentially expressed genes. A protein-protein interaction (PPI) network was constructed using the STRING database to identify the closely connected modules. To conduct functional enrichment analysis of the relevant genes, GO and KEGG pathway analyses were performed with Cluster Profiler. Pivot analysis of the ncRNAs and TFs was performed by using the RAID2.0 database and TRRUST v2 database. TF-mRNA regulatory relationships were analyzed in the TRRUST V2 database. Hubgene targeting relationships were screened in the TargetScan, miRTarBase and miRDB databases. The SNV data of the hub genes were analyzed by using the R maftools package. A ROC curve was drawn based on the TCGA database. The proportion of immune cells was estimated using CIBERSORT and the LM22 feature matrix. Results. The results showed that the matrix score was significantly correlated with colorectal cancer stage T. A total of 789 differentially expressed genes and 121 survival-related prognostic genes were identified. The PPI network showed that 22 core genes were related to the CRC prognosis. Furthermore, 4 ncRNAs that regulated the core prognosis genes, 11 TFs with regulatory effects on the core prognosis genes, and two drugs, quercetin and pseudoephedrine, that have regulatory effects on colorectal cancer were also identified.Conclusions. We obtained a list of tumor microenvironment-related genes for CRC patients. These genes could be useful for determining the prognosis of CRC patients. To confirm the function of these genes, 
1

2

3 1. Department of Gastrointestinal Surgery, The Affiliated Hospital of Jiaxing University, Jiaxing, Zhejiang,

5

6 Correspondence to: Yi Zhu: Department of Gastrointestinal Surgery, The Affiliated Hospital of Jiaxing

7 University, Jiaxing, Zhejiang, China.

8 Telephone: +8613967350889

9 Mail Address: zhuanwen456@163.com

10

11

12 


\section{Abstract}

Background. Colorectal cancer (CRC) is one of the most common malignancies. An early diagnosis and an accurate prognosis are major focuses of $\mathrm{CRC}$ research. Tumor microenvironment cells and the extent of infiltrating immune and stromal cells contribute significantly to the tumor prognosis.

Methods. Immune and stromal scores were calculated based on the ESTIMATE algorithm using the sample expression profile of the TCGA database. GSE102479 was used as the validation database. Differentially expressed genes whose expression was significantly associated with the prognosis of CRC patients were identified based on the immune matrix score. Survival analysis was conducted on the union of the differentially expressed genes. A protein-protein interaction (PPI) network was constructed using the STRING database to identify the closely connected modules. To conduct functional enrichment analysis of the relevant genes, GO and KEGG pathway analyses were performed with Cluster Profiler. Pivot analysis of the ncRNAs and TFs was performed by using the RAID2.0 database and TRRUST v2 database. TF-mRNA regulatory relationships were analyzed in the TRRUST V2 database. Hubgene targeting relationships were screened in the TargetScan, miRTarBase and miRDB databases. The SNV data of the hub genes were analyzed by using the R maftools package. A ROC curve was drawn based on the TCGA database. The proportion of immune cells was estimated using CIBERSORT and the LM22 feature matrix.

Results. The results showed that the matrix score was significantly correlated with colorectal cancer stage T. A total of 789 differentially expressed genes and 121 survival-related prognostic genes were identified. The PPI network showed that 22 core genes were related to the CRC prognosis. Furthermore, 4 ncRNAs that regulated the core prognosis genes, $11 \mathrm{TFs}$ with regulatory effects on the core prognosis genes, and two drugs, quercetin and pseudoephedrine, that have regulatory effects on colorectal cancer were also identified. could be useful for determining the prognosis of CRC patients. To confirm the function of these genes, additional experiments are necessary. 
55 Keywords Prognosis; Tumor microenvironment; Immune scores; Colorectal cancer

56

57

58

59

60

61

62

63

64

\section{Introduction}

Colorectal cancer (CRC) is a common malignant tumor [1]. Despite advances in understanding the molecular mechanisms of CRC, it still has a high mortality rate. Guided treatment based on outcome prediction is an effective method to reduce the mortality of CRC [2]. In recent years, studies on the prognostication of CRC have become increasingly important, and a large number of potential outcome predictors have been identified [3-4]. The pathogenesis of CRC is comprehensive. Tumor cell intrinsic genes, especially master transcription factors, dictate the initiation, progression, and evolution of CRC [5-6]. The tumor microenvironment has also been reported to critically influence the expression of genes in tumor tissues and the prognosis [7-8].

The tumor microenvironment is the cellular environment of tumor cells, consisting of extracellular matrix, soluble molecules and tumor stromal cells. In the tumor microenvironment, immune cells and stromal cells are the two main types of nontumor components and have been proposed to be valuable for the diagnosis and prognosis evaluation of tumors [9]. In 2011, scientists began to examine various stages (TNMI-IV) of CRC for immunity to authenticate grading and staging systems, and there was considerable research on immune scoring stages and TNM staging. The results showed that an immune scoring system could predict the outcomes of CRC more accurately. It has obvious advantages for predicting survival in patients with CRC [10-12]. According to the study conducted by Yoshihara et al. [13], immune and stromal scores could be used to predict the infiltration of nontumor cells by analyzing specific gene expression data from The Cancer Genome Atlas (TCGA) database of immune and stromal cells.

However, no research has been conducted on predicting the prognosis-related genes of CRC by using the tumor microenvironment score. For the first time, in this current work, by taking advantage of both the TCGA database of CRC cohorts and the ESTIMATE algorithm-derived immune scores, we extracted a list of microenvironment-associated genes that predict the outcomes of CRC patients.

\section{Materials \& Methods}

Materials

Peer] reviewing PDF | (2021:02:58414:3:0:NEW 29 Sep 2021) 
82 (1) Gene expression profile data and the clinical information of 362 CRC patients from TCGA were obtained 83 from the NCI Genomic Data Commons (https://portal.gdc.cancer.gov)[14], and samples with missing survival 84 information or a follow-up of fewer than 30 days were excluded (Table 1).

85 (2) Gene expression profile data and clinical information from the GSE102479 dataset (152 CRC patients)

86

87 were obtained from the Gene Expression Omnibus (GEO) database, and samples with missing survival information or a follow-up of fewer than 30 days were removed from the subsequent verification (Table 2).

\section{(1) Calculation of the immune matrix score and identification of differentially expressed genes (DEGs)}

The ESTIMATE algorithm was applied to calculate the immune score and stromal score of the sample expression profile of the TCGA CRC patients. Maxstat software was used to find the best cut-off of the immune score and matrix score. Samples were classified into two groups with high and low scores based on the score of the best cut-off to analyze and calculate the DEGs. Limma was used to analyze the gene expression data after processing, and $\log ($ Fold Change $)>0.2$ and $\mathrm{P}$ value $<0.05$ were taken as the standard to define the DEGs.

\section{(2) Survival analysis}

Survival analysis was conducted on the union of DEGs, and a Kaplan-Meier diagram was drawn to illustrate the relationship between the overall survival of patients and the expression level of the DEG genes. By using the log-rank test, the DEGs with $\mathrm{p}<0.05$ were defined as survival-related prognostic genes.

\section{(3) PPI network}

Survival-related prognostic genes were placed in the String database to retrieve the PPI network, identify the closely connected modules in the network, and define the closely connected module genes as the core prognostic genes.

\section{(4) Enrichment analysis}

Cluster Profiler was used to conduct functional enrichment analysis on relevant genes, and the Gene Ontology (GO) terms of significant enrichment were further identified according to the biological process (BP) functional enrichment analysis, while Kyoto Encyclopedia of Genes and Genomes (KEGG) pathway enrichment analysis was performed to identify the biological processes with significant enrichment. 
109 Enrichment analysis of KEGG pathways in GSEA was performed using the R package enrichplots for immune

110 and matrix groupings ( $\mathrm{P}$ value cut-off $=0.05$ ).

111 (5) Pivot analysis

112 The pivot node refers to 1) having at least two interactions with the module gene; 2) the significance analysis

113 of the interaction between the node and each module should be less than or equal to 0.05 , and the statistical

114 method is hypergeometric.

115 Pivot analysis method of the ncRNA: The ncRNA-mRNA interaction relationship included in the RAID2.0

116 database is the background of the interaction. All of the interactions between ncRNAs and module genes were

117 counted. Then, the interactions between each ncRNA and the in-module genes were counted.

118 Pivot analysis method of transcription factors (TFs): according to the interaction background of the human TF-

119 mRNA regulation in the TRRUST v2 database, all TF interactions with the module genes were counted, then

120 each TF interaction with the in-module genes and out-module genes were counted, and the pivot was screened

121 according to the significance of the $\mathrm{p}$ value of the hypergeometric test.

122 (6) Analysis of the hub genes

123 Target gene-TF regulatory network: Human TF-mRNA regulatory relationships were downloaded from the

124 TRRUST V2 database to screen the 22 transcription factors that interacted with the hub genes.

125 Target gene-miRNA regulatory network: Human miRNA-mRNA targeting relationships were downloaded

126 from the TargetScan (http://www.targetscan.org/vert_72/), miRTarBase database

127 (http://mirtarbase.mbc.nctu.edu.tw/php/index.php) and miRDB database (http://mirdb.org/). Based on the

128 human miRNA-mRNA targeting relationships in the TargetScan, miRTarBase and miRDB databases, 22 hub

129 gene targeting relationships were screened.

130 Mutation analysis of the hub genes: The R maftools package was used to analyze the single nucleotide 131 variation (SNV) data of the hub gene samples obtained from TCGA.

132 The receiver operating characteristic (ROC) curve based on the hub genes: Based on the TCGA dataset, 133 ROC curves were drawn for hub genes with a predict.time value of 1095.

134 Immune infiltration analysis of the hub genes: The proportion of immune cells in the colorectal cancer 135 samples was estimated using the CIBERSORT and LM22 feature matrices. The Pearson correlation coefficient 
136 of the hub gene and the proportion of infiltration of the immune cells were calculated using the Psych and ar 137 packages.

\section{Results}

140 (1) The matrix score was significantly correlated with stage T colorectal cancer

141 CRC expression profile data were downloaded from the TCGA database. Clinical information (OS $>1$ month) 142 and immune and stromal scores calculated by the ESTIMATE algorithm were integrated. In addition, 362 143 CRC samples were finally retained for subsequent analysis. The distribution of the immune score and stromal score is shown in Figure 1A. To determine the potential correlation between the overall survival rate and the immune and stromal scores, Maxstat software was used to find the optimal cut-offs for the immune and stromal scores. Cancer samples were divided into high and low scores according to the score of the optimal cut-off (stromal score=-207.8886/immune score=-708.0627), and the corresponding clinical information was used for survival analysis. In the survival curve of the immune score and matrix score, we found that the immune score and matrix score were significantly correlated with the survival time of the patients $(\mathrm{p}=0.0035 / \mathrm{p}=0.0034$, Figure $1 \mathrm{~B}-1 \mathrm{C})$. At the same time, the results showed that the stromal score had no statistical significance with all stages, but it was significantly correlated with $\mathrm{T}$ staging in the correlation analysis $(\mathrm{p}=0.28 / \mathrm{p}=0.029$, Figure $1 \mathrm{D}-1 \mathrm{E})$. There was no statistical significance in the correlation between the immune score and staging. However, there was a certain difference in the scores for each stage $(\mathrm{p}=0.34 / \mathrm{p}=0.93$, Figure $1 \mathrm{~F}-1 \mathrm{G})$.

\section{(2) Comparison of colorectal cancer gene expression with immune score and stromal score}

To reveal the correlations between gene expression and the immune and matrix scores, we evaluated the expression profile data of CRC patients in the TCGA database. A total of 228 DEGs were identified according to the matrix score (100 cases with a high score and 262 cases with a low score), among which 163 genes were upregulated and 65 genes were downregulated. The results showed that some of the DEGs were significantly different between the two groups (Figure 2A). A total of 579 DEGs were identified according to the immune score groups (38 cases with high scores and 324 cases with low scores), among which 301 genes were upregulated and 278 genes were downregulated. These 789 DEGs were used as key genes for subsequent 
163

164

165

166

167

168

169

170

171

172

173

174

175

176

177

178

179

180

181

182

183

184

185

186

187

188

189

analysis (Figure 2B), and their details are listed in Table s1. To clarify the potential function of the 789 DEGs, GO and KEGG enrichment analyses were performed on the DEGs. The results are shown in Figure 3A and Figure 3B (the top 8 were selected for display). GO analyses revealed that these DEGs were mostly involved in tissue-specific immune responses, positive regulation of adaptive immune responses and so on. In addition, according to the KEGG pathway analyses, the DEGs were involved in the age-race signaling pathway in diabetic complications, cytokine-cytokine receptor interaction, IL17 signaling pathway and so on. The results of the enrichment analysis of KEGG pathways in GSEA showed that the genes were mostly enriched in the pathways of asthma, graft-versus-host disease, malaria, rheumatoid arthritis, and viral protein interaction with cytokines and cytokine receptors based on the ImmuneScore (Figure 4A). In addition, the results showed that the genes were mostly enriched in the ECM-receptor interaction, hypertrophic cardiomyopathy, protein digestion and absorption, Staphylococcus aureus infection, and systemic lupus erythematosus pathways based on the Stromalscore (Figure 4B).

\section{(3) Survival analysis of DEGs}

To screen out the genes associated with the prognosis of CRC, we divided the 789 DEGs into high and low expression groups according to their median expression and performed survival analysis. A total of 121 survival-related prognostic genes were extracted $(\mathrm{p}<0.05)$. The survival curves of some of the prognostic genes are shown in Figure 5A.

\section{(3) Construction of PPI networks between genes}

To better understand the interactions between the identified prognostic genes, we used the STRING database to obtain a PPI network consisting of 35 nodes and 36 edges (Figure 6). We identified tightly linked modules (green parts) in the network and defined the tightly coupled module genes as the core prognostic genes $(\mathrm{N}=22)$. GO analysis and KEGG enrichment analysis were performed on 22 core prognostic genes. The results of the enrichment analysis are shown in Figure 7A-7B (the top 10 genes were selected for display).

\section{(5) Identification of the core prognostic genes}

The 22 core prognostic genes obtained were validated in the GEO dataset, and all 22 genes were expressed normally. Survival analysis of the 22 core prognostic genes was performed in this dataset and identified only one gene consistent with the TCGA results $(\mathrm{P}<0.05)$. The results of the gene survival analysis are shown in 
190 Figure 5B.

191

192

193

194

195

196

197

198

199

200

201

202

203

204

205

206

207

208

209

210

211

212

213

214

215

216

(6) Regulation of the core prognostic genes by $n c R N A / T F$

Based on the 51913 ncRNA-mRNA interaction relationship in the RAID2.0 database, we searched for the pivot node (ncRNA) of the regulatory function module. When the $\mathrm{p}$ value $<0.05$, we obtained four ncRNAs that regulated the core prognosis gene (Table 3), including metastasis-associated lung adenocarcinoma transcript 1 (MALAT1) (associated with tumor cell proliferation and metastasis), colorectal neoplasia differentially expressed (CRNDE) (promotes colorectal cancer cell proliferation), FOXF1 adjacent noncoding developmental regulatory RNA (FENDRR) and taurine upregulated gene 1 (TUG1) (regulation of resistance to colorectal cancer methotrexate).

Based on the interaction relationships of 9396 human TF-mRNAs contained in the TRRUST v2 database, the pivot node (TF) of the regulatory function module was searched. When $\mathrm{p}$ value $<0.05,11 \mathrm{TFs}$ with regulatory effects on the core prognostic genes were obtained (Table 4), including a number of cancer-related transcription factors, such as activating transcription activator 1 (ATF1), CCAAT/enhancer binding proteins C/EBP beta (CEBPB), E2F transcription Factor 3 (E2F3), ETS translocation variant 4 (ETV4), and Y-box protein 1 (YBX1). The visualization of the core prognostic genes and ncRNA/TF interactions is shown in Figure 8A.

(7) Target gene-TF regulatory network of the 22 hub genes

Human TF-mRNA regulatory relationships were downloaded from the TRRUST V2 database to screen for transcription factors interacting with the 22 hub genes, including $M Y B, F O X D 3$, NFKB1, etc. (Figure 8B).

(8) Target gene-miRNA regulatory network of the 22 hub genes

A total of 21363630 human miRNA-mRNA targeting relationships were downloaded from TargetScan. A total of 502652 human miRNA-mRNA targeting relationships were downloaded from the miRTarBase database. A total of 1102737 human miRNA-mRNA targeting relationships were downloaded from the miRDB database. Based on the human miRNA-mRNA targeting relationships in the TargetScan, miRTarBase and miRDB databases, 22 hub gene targeting relationships were screened, and 81 pairs of targeting relationships existed in all three databases (Figure 8C).

(9) Mutation analysis of the hub genes

Peer] reviewing PDF | (2021:02:58414:3:0:NEW 29 Sep 2021) 
217 As shown in Figure 9, the 22 hub gene samples had mutations only in 90 samples. Among these, ERBB2 had

218 the highest mutation rate, most of which were missense mutations, and most of the samples had mutations of $219 \mathrm{C}>\mathrm{T}$.

220 (10) The ROC curve based on the hub genes

221 The area under the curve (AUC) of the 22 hub genes ranged from 0.344 to 0.669 . Twelve hub genes,

222

223

224

225

226

227

228

229

230

231

232

233

234

235

236

237

238

239

240

241

242

243

ENO2, GRP, HIST1H2AC, HIST1H2AK, HIST1H4H, HIST4H4, HOXB4, HSPB1, MIFT, POMC, QRFP, and TNFAIP6, had weak value in predicting the survival of colorectal cancer with $>0.5$ AUCs (Figure 10).

(11) Immune infiltration analysis of the hub genes

As shown in Figure 11, most of the hub genes were significantly correlated with plasma cells, M1 macrophages and other immune cells.

\section{(12) Exploration of drugs for the treatment of colorectal cancer}

For the core prognostic genes and related regulatory factors, we used the hypergeometric distribution test to screen related drugs in the context of drug-gene interactions in DrugBank. Finally, two markers of CRC regulation were obtained (Table 5). Among them, quercetin can significantly inhibit the action of cancerpromoting agents, inhibit the growth of isolated malignant cells, and inhibit the DNA, RNA and protein synthesis of Ehrlich ascites cancer cells.

\section{Discussion}

In the current work, we identified tumor microenvironment-related genes that contribute to CRC overall survival in the TCGA database and found that both the immune score and matrix score were significantly correlated with the patient survival time. Importantly, we were able to validate 789 DEGs. In addition, 121 prognostic genes were found to be associated with survival. Twenty-two genes were identified as core prognostic genes. Four ncRNAs that regulate the core prognostic genes, 11 TFs that had a regulatory effect on the core prognostic genes, and 2 drugs that have regulatory effects on CRC were also identified.

Both the immune score and the matrix score can predict the purity of the tumor, as well as the number of stromal and immune cells. The more stromal and immune cells there are, the lower the purity of the tumor. In a study of immune scoring by Galon et al., patients with early TNM (stage I and II) were followed up for 
244

245

246

247

248

249

250

251

252

253

254

255

256

257

258

259

260

261

262

263

264

265

266

267

268

269

270 survival and recurrence of colon cancer, and it was found that patients with high immune scores had a longer survival period. Ninety-five percent of patients with a high score had no tumor recurrence within 18 years after surgery. Fifty percent of patients with low scores had a recurrence within 2 years after surgery [15]. In 2018, Pages et al. reported that immune scores could provide a reliable assessment of the risk of recurrence of colon cancer, which supports the use of immune scores as part of the new TNM immunotyping [16]. Therefore, as a perfect supplement to the TNM staging system, the immune score will be a superior staging system for predicting the survival of tumor patients.

Searching for more metastatic genes related to CRC and studying their biological characteristics and the specific mechanism of metastasis can help control tumor metastasis, prevent recurrence and improve the prognosis of patients. In terms of multigene early diagnosis and a prognostic model of CRC, Marshall et al. obtained an early diagnostic model of CRC based on the gene expression profile of the peripheral blood of CRC patients [17]. Sveen et al. proposed a prognostic model by studying gene expression in tumor tissues, which has potential prognostic value in stage IV and V CRC patients [18]. In addition, a large number of genes, including P53 [19], K-ras [20], B-raf [21], and N-ras [22], have been shown to be associated with the prognosis of CRC.

In the present study, 22 genes were identified as the core prognostic genes of CRC. Among them, $M A P K 8$ [23], HSPB1 [24], ILIB [25], PRIM1 [26] and so on were shown to be associated with the susceptibility to or prognosis of CRC. However, only the PRIM1 gene was validated in the GEO database.

PRIM1 (primase) plays a key role in the process of DNA synthesis initiation by synthesizing RNA primers for Okazaki fragments [26]. Mutations in PRIM1 cause extensive apoptosis of retinal neurons through activation of the DNA damage checkpoint and tumor suppressor p53[27]. PRIM1 plays an important role in the strict control of the DNA replication fork during tumor cell proliferation and was shown to be involved in estrogen-induced breast cancer formation through activation of the G2/M cell cycle checkpoint [28]. In addition, PRIM1 [29] has been reported to be associated with CRC progression, and this gene is likely to be a prognostic marker of the CRC immune microenvironment. However, the mechanism by which PRIM1 affects CRC is not clear.

A total of 4 core ncRNAs were obtained that could regulate the core prognostic genes in the present study.

Peer] reviewing PDF | (2021:02:58414:3:0:NEW 29 Sep 2021) 
271 MALAT1 is a long noncoding RNA with important biological functions and is located on chromosome llq13.1

272 [30]. MALT1 expression is upregulated in CRC tumor cells. A-kinase anchoring protein 9 (AKAP9) is a target

273 gene for the cancer-promoting effect of MALAT1. AKAP9 is related to the polarization of cell spinning

274 chains, and the overexpression of MALAT1 in normal intestinal epithelial cells causes them to divide and

275 differentiate and promotes tumorigenesis [31]. In vitro experiments have demonstrated that its knockout can

276 inhibit the migration of p-catenin from the cytoplasm to the nucleus, resulting in decreased expression of c-

277 MYC and MMP-7, whereas overexpression of MALAT1 can activate the Wnt/p-catenin pathway and promote

278 the invasion and metastasis of CRC [32]. Zheng et al. further confirmed that MALAT1 was significantly

279 upregulated in CRC tissues and was associated with a poor prognosis in stage II/III CRC patients [33].

$280 C R N D E$ was first identified as a biomarker of CRC. High expression of IncRNA CRNDE was detected in

281 more than $90 \%$ of colorectal adenomas and adenocarcinoma cells [34]. Experiments showed that the regulatory

282 effect of $C R N D E$ was related to the IGF signaling pathway and was involved in the carcinogenic process of

283 intestinal epithelial cells [35]. CRNDE can activate the $\mathrm{Wnt} / \mathrm{x}$-catenin signaling pathway in CRC patients, thus

284 promoting tumor cell proliferation, invasion and other processes [36]. In addition, Li et al. showed that

$285 C R N D E$ can be used as a potential noninvasive serum marker to predict the efficacy of first-line FOLFOX

286 regimens for metastatic colorectal cancer and it is associated with a poor prognosis [37]. Although $C R N D E$ can

287 enhance the proliferation, migration and invasion of CRC cell lines, the mechanism by which CRNDE

promotes the proliferation and migration of CRC cells remains unclear.

Taurine upregulated gene 1 (TUGl) is a newly discovered carcinogenic lncRNA located on chromosome 22q12 [38]. Numerous studies have demonstrated that TUGl expression levels are significantly elevated in

CRC tissues and cell lines. The expression level of $T U G l$ in CRC tissues was 4-6 times higher than that in adjacent noncancerous tissues [39]. In vitro tests, overexpression of $T U G l$ can induce the formation of tumor cell colonies and activate the expression of EMT-related genes to improve the invasion and metastasis of tumors and promote liver metastasis of CRC [40]. TUGl promotes cancer cell proliferation, migration, invasion, and epithelial-mesenchymal transition (EMT) and inhibits tumor cell apoptosis through complex mechanism of $T U G l$ remains to be further elucidated [41]. 
Transcription factors activate the transcription of downstream target genes by binding to the target gene promoter region. Studies have found that transcription factors are closely related to tumors. A total of $11 \mathrm{TF}$ genes regulating the core prognosis were obtained in the present study. We are particularly interested in ATF1, $C E B P B, E 2 F 3$, and $E T V 4$. ATF1 belongs to the cAMP response element binding protein (cyclic AMP response element binding protein, after CREB) family [41]. A study found that ATF1 in a wide variety of tumors plays a role as both an oncogene and a tumor suppressor gene by influencing the cell signal transduction pathways involved in tumor cells related to biological processes, such as proliferation, apoptosis, angiogenesis, migration, invasion, and immune surveillance, which affects the occurrence and development of tumors [42]. In mouse colorectal cancer cells, active phosphorylated CREB is elevated [43], and p300 can assist the phosphorylation of CREB and activate intestinal stem cell transcription factors, including Myb, that regulate the proliferation of intestinal epithelial cells [44]. It was speculated that $A T F 1$ is involved in human colorectal cancer cell proliferation through the p300-MYB-CREB axis.

The transcription factor $C E B P B$ is involved in a number of biological processes, including cell differentiation, metabolic balance, proliferation, tumorigenesis, apoptosis, immune and stress responses, energy metabolism, and blood production [45]. Some studies have shown that CEBPB affects tumorigenesis by interacting with other genes to form a regulatory network during tumorigenesis and development [46]. Other studies have also shown that the LIP subtype of the C/EBP transcription factor can induce apoptosis of human breast cancer cells and induce its own phagocytosis, which may be acting as a tumor suppressor by inducing tumor autophagy [47].

Additionally, the $E 2 F$ transcription factor is an important regulator of G1 phase entry into S phase in the cell cycle, and it is closely related to tumor occurrence and cell apoptosis [48]. Hurst et al. [49] showed that E2F3 inhibited pRb and P53 through two important pathways of cell proliferation regulation and tumor monitoring, p16(Ink4a)-cycd/cdk4-rb-e2f and Arf/mdm2-p53, respectively, leading to the disorder of cell cycle regulation and thus promoting the occurrence and development of tumors. Wang et al. found that the expression of $E 2 F 3$ was high in colorectal carcinoma. There is a positive correlation with the expression of $E 2 F 3$ in colorectal carcinoma [50].

Furthermore, ETV4 is a member of the polyomavirus enhancer activator 3 (PEA3) subfamily of ETS 
325

326

327

328

329

330

331

332

333

334

335

336

337

338

339

340

341

342

343

344

345

346

347

348

349

350

351 transcription factors, in which transcription factors can recognize and bind to GGAA/T sequences to regulate the expression of multiple target genes, thus affecting the occurrence and development of diseases. Existing studies have found that ETV4's ability to promote cancer migration is closely related to MMP [51]. For example, in breast cancer, ETV4 can promote the migration of cancer cells by promoting the expression of MMP2 [52]; in esophageal cancer, ETV4 can promote the metastasis of cancer cells by promoting the expression of MMP1 [53]. In addition, ETV4 was found to promote the invasion and metastasis of cancer cells by promoting the greening of $\mathrm{COX} 2$ [54]. Of course, the mechanisms of the pro-proliferative and promigratory effects of ETV4 in CRC are not fully understood.

Notably, we also predicted that quercetin might be a drug that regulates CRC. Quercetin is a flavonoid with a wide range of biological activities, including antioxidant activity, scavenging oxygen free radicals, antifibrosis activity, lowering blood pressure, lowering blood glucose, protecting heart muscle and antitumor activity [55]. In recent years, many studies have found that quercetin can inhibit a variety of cancer cells, especially colon cancer. Quercetin can not only inhibit the proliferation and induce the apoptosis of colon cancer cells but also reduce the number of abnormal gland crypts in the colon [56]. However, there are few studies on the effect of quercetin on colon cancer, and the specific molecular mechanism is still not fully understood. Luo et al. reported that quercetin may inhibit cell proliferation and induce apoptosis through the bcl-2 and c-myc genes [57]. Park et al. [58] found that quercetin could significantly inhibit the transcriptional activity of catenin/Tcf signals in SW480 colon cancer cells by reducing the protein levels of large-catenin and Tef in the nucleus. Thus, quercetin can be used as an adjuvant drug to inhibit the growth of colon cancer cells and has potential value in the drug treatment of colon cancer.

\section{Conclusions}

In summary, from the functional enrichment analysis of the TCGA database applied by ESTIMATE algorithm-based immune scores, we extracted a list of tumor microenvironment-related genes. These genes could be useful for determining the prognosis of CRC patients. In addition, further investigation of these genes as well as their regulators, including ncRNAs and TFs, provides a stronger predictor of survival than individual genes. Finally, the detection of drugs that regulate CRC could lead to novel insights into potential treatments

Peer] reviewing PDF | (2021:02:58414:3:0:NEW 29 Sep 2021) 
352 for CRC.

353

354 Acknowledgments: None.

355 Funding: This work was supported by the Basic Public Welfare Research Program of Zhejiang Province, 356 China (LGF18H160033); the Medical and Health Science and Technology Project of Zhejiang Province, 357 China(2019KY214); the Medical and Health Science and Technology Project of Zhejiang Province, China 358 (2019KY692); Project of Public Welfare research of Jiaxing (2019AD32257); the Jiaxing Key Discipiline of 359 Medcine-Oncology (Supporting Subject) (2019-zc-11).

360 Competing Interests The authors declare that they have no competing interests.

361

362

363

364

365

366

367

368

369

370

371

372

373

374

375

376

377

378

\section{References}

1. Long AG, Lundsmith ET, Hamilton KE. Inflammation and Colorectal Cancer. Current Colorectal Cancer Reports. 2017; 13(4): 341-351.

2. Yu J, Feng Q, Wong SH, Zhang D, Liang QY, Qin Y, Tang L, Zhao H, Stenvang J, Li Y, Wang X, Xu X, Chen $\mathrm{N}, \mathrm{Wu}$ WK. Metagenomic analysis of faecal microbiome as a tool towards targeted non-invasive biomarkers for colorectal cancer. Gut. 2017; 66(1): 70-78.

3. Sanz-Garcia E, Argiles G, Elez E, Tabernero J. BRAF mutant Colorectal Cancer: prognosis, treatment and new perspectives. Annals of Oncology. 2017; 28(11): 2648-2657.

4. Rena O, Casadio C, Viano F, Cristofori R, Ruffini E, Filosso PL, Maggi G. Pulmonary resection for metastases from colorectal cancer: factors influencing prognosis. Twenty-year experience. 2002; 21(5): 906912.

5. Wang Q, Hu B, Hu X, Kim H, Squatrito M, Scarpace L, deCarvalho AC, Lyu S, Li P, Li Y, Barthel F, Cho HJ, Lin YH. Tumor Evolution of Glioma-Intrinsic Gene Expression Subtypes Associates with Immunological Changes in the micro-environment. Cancer Cell. 2017; 32: 42-56.e6.

6. Suvà ML, Rheinbay E, Gillespie SM, Patel AP, Wakimoto H, Rabkin SD, Riggi N, Chi AS, Cahill DP, Nahed BV, Curry WT, Martuza RL, Rivera MN. Reconstructing and reprogramming the tumor-propagating potential of glioblastoma stem-like cells. Cell. 2014; 157: 580-594. 
379

380

381

382

383

384

385

386

387

388

389

390

391

392

393

394

395

396

397

398

399

400

401

402

403

404

405

7. Curry JM, Sprandio J, Cognetti D, Luginbuhl A, Bar-ad V, Pribitkin E, Tuluc M. Tumor micro-environment in head and neck squamous cell carcinoma. Semin Oncol. 2014; 41: 217-234.

8. Cooper LA, Gutman DA, Chisolm C, Appin C, Kong J, Rong Y, Kurc T, Van Meir EG, Saltz JH, Moreno $\mathrm{CS}$, Brat DJ. The tumor micro-environment strongly impacts master transcriptional regulators and gene expression class of glioblastoma. Am J Pathol. 2012; 180: 2108-2119.

9. Fukumura D, Duda DG, Munn LL, Jain RK. Tumor Microvasculature and micro-environment: Novel Insights Through Intravital Imaging in Pre-Clinical Models. Microcirculation. 2010; 17(3): 206-225.

10. Mlecnik B, Bindea G, Franck Pagès, Galon J. Tumor immunosurveillance in human cancers. Cancer \& Metastasis Reviews. 2011; 30(1): 5-12.

11. Fridman WH, Franck P, Catherine SF, Galon J. The immune contexture in human tumours: impact on clinical outcome. Nature Reviews Cancer. 2012; 12(4): 298-306.

12. Galon J. Type, Density, and Location of Immune Cells Within Human Colorectal Tumors Predict Clinical Outcome. Science. 2006; 313(5795): 1960-1964.

13. Yoshihara K, Shahmoradgoli M, Martínez E, Vegesna R, Kim H, Torres-Garcia W, Treviño V, Shen H, Laird PW, Levine DA, Carter SL, Getz G, Stemke-Hale K. Inferring tumour purity and stromal and immune cell admixture from expression data. Nat Commun. 2013; 4: 2612.

14. Heath AP, Ferretti V, Agrawal S, An M, Angelakos JC, Arya R. The NCI Genomic Data Commons. Nat Genet. 2021; 53: 257-262.

15. Galon J, Pagès F, Marincola FM, Angell HK, Thurin M, Lugli A, Zlobec I, Berger A, Bifulco C, Botti G, Tatangelo F, Britten CM, Kreiter S. Cancer classification using the Immunoscore: a worldwide task force. J Transl Med. 2012; 10(1): 205.

16. Pagès F, Mlecnik B, Marliot F, Bindea G, Ou FS, Bifulco C, Lugli A, Zlobec I, Rau TT, Berger MD, Nagtegaal ID, Vink-Börger E, Hartmann A. International validation of the consensus Immunoscore for the classification of colon cancer: a prognostic and accuracy study. Lancet. 2018; 391(10135): 2128-2139.

17. Marshall KW, Mohr S, Khettabi FE, Nossova N, Chao S, Bao W, Ma J, Li XJ, Liew CC. A blood-based biomarker panel for stratifying current risk for colorectal cancer. Int J Cancer. 2010; 126 (5): $1177-1186$.

18. Sveen A, Agesen TH, Nesbakken A, Meling GI, Rognum TO, Liestøl K, Skotheim RI, Lothe RA. 
406 ColoGuidePro: A Prognostic 7-Gene Expression Signature for Stage III Colorectal Cancer Patients. Clinical

407 Cancer Research An Official Journal of the American Association for Cancer Research. 2012; 18(21): 60014086010

409 19. Lüchtenborg M, Weijenberg MP, Roemen GM, de Bruïne AP, van den Brandt PA, Lentjes MH, Brink M, 410 van Engeland M, Goldbohm RA, de Goeij AF. APC mutations in sporadic colorectal carcinomas from The 411 Netherlands Cohort Study. Carcinogenesis. 2004; 25(7): 1219-1226.

412 20. Andreyev HJN, Norman AR, Clarke PA, Oates JR, Clarke PA. Kirsten ras Mutations in Patients With 413 Colorectal Cancer: the Multicenter \"RASCALl" Study. Journal of the National Cancer Institute. 1990; 90(9): $414 \quad 675-684$.

415 21. De Roock W, Claes B, Bernasconi D, De Schutter J, Biesmans B, Fountzilas G, Kalogeras KT, Kotoula V, 416 Papamichael D, Laurent-Puig P, Penault-Llorca F, Rougier P, Vincenzi B, Santini D, Tonini G, Cappuzzo F. 417 Effects of KRAS, BRAF, NRAS, and PIK3CA mutations on the efficacy of cetuximab plus chemotherapy in 418 chemotherapy-refractory metastatic colorectal cancer: a retrospective consortium analysis. Lancet Oncol. $4192010 ; 11(8): 753-762$.

420 22. Parsons MT, Buchanan DD, Thompson B, Young JP, Spurdle AB. Correlation of tumour BRAF mutations 421 and MLH1 methylation with germline mismatch repair (MMR) gene mutation status: a literature review 422 assessing utility of tumour features for MMR variant classification. J Med Genet. 2012; 49(3): $151-157$.

423 23. Slattery ML, Lundgreen A, Wolff RK. MAP kinase genes and colon and rectal cancer. Carcinogenesis. $424 \quad 33(12): 2398-2408$.

425 24. Nadin SB, Cuello-Carrión FD, Sottile ML, Ciocca DR, Vargas-Roig LM. Effects of hyperthermia on 426 Hsp27 (HSPB1), Hsp72 (HSPA1A) and DNA repair proteins hMLH1 and hMSH2 in human colorectal cancer 427 hMLH1-deficient and hMLH1-proficient cell lines. International Journal of Hyperthermia the Official Journal 428 of European Society for Hyperthermic Oncology North American Hyperthermia Group. $2012 ; 28(3)$ : $191-201$.

429 25. Sanabria-Salas MC, Hernández-Suárez G, Umaa-Pérez A, Tenesa A, Serrano-López ML, de Gómez MS, 430 Rojas MP, Bravo LE, Albis R, Plata JL, Green H, Borgovan T, Li L, Majumdar S, Garai J, Lee E. IL1B-CGTC 431 haplotype is associated with colorectal cancer in admixed individuals with increased African ancestry. 432 Scientific Reports. 7: 41920. 
433

434

435

436

437

438

439

440

441

442

443

444

445

446

447

448

449

450

451

452

453

454

455

456

457

458

459

26. Cloutier S, Hamel H, Champagne M, Yotov WV. Mapping of the human DNAprimase 1 (PRIM1) to chromosome 12q13. Genomics 1997; 43: 398-401.

27. Yamaguchi M, Fujimori-Tonou N, Yoshimura Y, Kishi T, Okamoto H, Masai I. Mutation of DNA primase causes extensive apoptosis of retinal neurons through the activation of DNA damage checkpoint and tumor suppressor p53. Development. 2008; 135: 1247-1257.

28. Lee WH, Chen LC, Lee CJ, Huang CC, Ho YS, Yang PS, Ho CT, Chang HL, Lin IH, Chang HW, Liu YR, Wu CH, Tu SH. DNA primase polypeptide 1 (PRIM1) involves in estrogen-induced breast cancer formation through activation of the G2/M cell cycle checkpoint. International Journal of Cancer. 2018; 31788.

29. Job A, Schmitt LM, Wenserski LV, Brigitte LB, Gress TM, Buchholz M, Gallmeier E. Inactivation of PRIM1 Function Sensitizes Cancer Cells to ATR and CHK1 Inhibitors. Neoplasia. 2018; 20(11): $1135-1143$.

30. Zhang B, Mao YTS, Diermeier SD, Eddy SR, Sanbonmatsu KY, Spector DL. Identification and Characterization of a Class of MALAT1 -like Genomic Loci. Cell Reports. 2017; 19(8):m1723-1738.

31. Xu C, Yang M, Tian J, Wang X, Li Z. MALAT-1: a long non-coding RNA and its important 3' end functional motif in colorectal cancer metastasis. Int J Oncol. 2011; 39 (1): 169-75.

32. Ji Q, Liu X, Fu XL, Zhang L, Sui H, Zhou L, Sun J, Cai J, Qin J, Ren J, Li Q. Resveratrol Inhibits Invasion and Metastasis of Colorectal Cancer Cells via MALAT1 Mediated Wnt/ $\beta$-Catenin Signal Pathway. Plos One. 2013; 8(11): e78700.

33. Zheng HT, Shi DB, Wang YW, Li XX, Xu Y, Tripathi P, Gu WL, Cai GX, Cai SJ. High expression of lncRNA MALAT1 suggests a biomarker of poor prognosis in colorectal cancer. International journal of clinical and experimental pathology. 2014; 7(6): 3174-3181.

34. Ning S, Zhao Z, Ye J, Wang P, Zhi H, Li R, Wang T, Li X. LincSNP: a database of linking diseaseassociated SNPs to human large intergenic non-coding RNAs. BMC Bioinformatics. 2014; 15(1): m152-158.

35. Huan J, Xing L, Lin Q, Xui H, Qin X. Long noncoding RNA CRNDE activates Wnt/ $\beta$-catenin signaling pathway through acting as a molecular sponge of microRNA-136 in human breast cancer. American Journal of Translational Research. 2017; 9(4): 1977.

36. Clevers H, Nusse R. Wnt/ $\beta$-Catenin Signaling and Disease. Cell. 2012; 149(6): 1192-1205.

37. Li YX, Wenwei H, Jun L, Xiong Q. The predictive value of serum lncRNA CRNDE for efficacy of first-

Peer] reviewing PDF | (2021:02:58414:3:0:NEW 29 Sep 2021) 
460 line FOLFOX chemotherapy in patients with metastatic colorectal cancer and their prognosis. Oncology 461 Progress. 2018; 7(16): 897-900.

462 38. Li Z, Shen J, Chan MTV, Wu WK. TUG1: a pivotal oncogenic long non-coding RNA of human cancers. 463 Cell Proliferation. 2016; 49(4): 471-475.

464 39. Sun J, Ding C, Yang Z, Liu T, Zhang X, Zhao C, Wang J. The long non-coding RNA TUG1 indicates a 465 poor prognosis for colorectal cancer and promotes metastasis by affecting epithelial-mesenchymal transition. 466 Journal of Translational Medicine. 2016; 14(1): 42.

467 40. Sun JF, Hu JY, Wang GJ, Yang Z, Zhao CL, Zhang XF, Wang JX. LncRNA TUG1 promoted KIAA1199 468 expression via miR-600 to accelerate cell metastasis and epithelial-mesenchymal transition in colorectal 469 cancer. Journal of Experimental \& Clinical Cancer Research. 2018; 37(1):106.

470 41. Huang GL, Liao D, Chen H, Lu Y, Chen L, Li H, Li B, Liu W, Ye C, Li T, Zhu Z, Wang J, Uchida T. 471 The protein level and transcription activity of activating transcription factor 1 is regulated by prolyl isomerase 472 Pin1 in nasopharyngeal carcinoma progression. Cell Death Dis. 2016; 7(12) : e2571.

473 42. Pu X, Storr SJ, Ahmad NS. High nuclear MSK1 is associated with longer survival in breast cancer patients. 474 J Cancer Res Clin Oncol. 2018; 144(3) : 509-517.

475 43. Sampurno S, Bijenhof A, Cheasley D, Xu H, Robine S, Hilton D, Alexander WS, Pereira L, 476 Mantamadiotis T, Malaterre J, Ramsay R. The Myb-p300-CREB axis modulates intestine homeostasis, 477 radiosensitivity and tumorigenesis. Cell Death Dis. 2013; 4: e605.

478 44. Ramaswamy K, Forbes L, Minuesa G, Gindin T, Brown F, Kharas MG, Krivtsov AV, Armstrong SA, Still 479 E, de Stanchina E, Knoechel B, Koche R, Kentsis A. Peptidomimetic blockade of MYB in acute myeloid 480 leukemia. Nat Commun. 2018; 9(1): 110.

481 45. Ramji D, Foka P. CCAAT/Enhancer Binding Proteins: Structure, Function and Regulation. Biochemical 482 Journal. 2002; 365(3):m561-575.

483 46. Abreu MM, Sealy L. The C/EBPbeta isoform, liver-inhibitory protein (LIP), induces autophagy in breast 484 cancer cell lines. Experimental Cell Research. 2010; 316(19): 3227-3238.

485 47. Koslowski M, Tureci O, Biesterfeld S, Seitz G, Huber C, Sahin U. Selective Activation of Trophoblast486 specific PLAC1 in Breast Cancer by CCAAT/Enhancer-binding Protein (C/EBP) Isoform 2. Journal of 
487

488

489

490

491

492

493

494

495

496

497

498

499

500

501

502

503

504

505

506

507

508

509

510

511283.

512 57. Luo HY, Feng DD, Yu W, Ding CB, Li J, Feng JH. Effects of Quercetin on Cell Proliferation and

513 Expression of Bcl-2 and C-myc in Colorectal Cancer Cell Line. China Pharmacy. 2014; 11: 164-171. 
514 58. Park $\mathrm{CH}$, Chang JY, Hahm ER, Park S, Kim HK, Yang CH. Quercetin, a potent inhibitor against $\beta$ 515 catenin/Tcf signaling in SW480 colon cancer cells. Biochem Biophys Res Commun. 2005; 328(1): 227-234.

516

517 Figure legends:

518 Figure 1. The score distribution and the Kaplan-Meier survival curves and the correlation of immune score and 519 stromal score and the stage of CRC A: the distribution of immune score and stromal score. B: Kaplan-Meier 520 survival curve based on stromal score. C: Kaplan-Meier survival curve based on immune score. D. The 521 correlation of stromals core and the stage of CRC. E. The correlation of stromal score and the stage T of CRC. 522 F. The correlation of immune score and the stage of CRC. G. The correlation of immune score and the stage T 523 of CRC.

Figure 2. The heat map and venn diagram of ifferentially expressed genes. A. Heat map of differential gene expression in gene score. B. Venn diagram. 579 differentially expressed genes were identified according to the immune score groups (38 cases with high score and 324 cases with low score), among which 301 genes were up-regulated and 278 genes were down-regulated.

Figure 3. The KEGG and GO analysis of differentially expressed genes. A. KEGG analysis of the 789 differentially expressed genes (DEGs). B. GO Biological Process of the 789 differentially expressed genes (DEGs).

Figure 4. The results of the enrichment analysis of KEGG pathway in GSEA. A. Enrichment analysis based on immunescore. B. Enrichment analysis based on stromalscore. gene (TCGA database). A total of 121 survival-related prognostic genes were extracted $(\mathrm{p}<0.05)$. B. KaplanMeier survival curve of the PRIM1 gene (GEO database). 
541 Figure 6. PPI network. The PPI network consisting of 35 nodes and 36 edges. A total of 22 core genes that 542 associated with CRC prognosis were defined.

543

544 Figure 7. The KEGG and GO analysis of 22 core prognostic genes. A. KEGG analysis of the 22 core 545 prognostic genes. B. GO Biological Process of the 22 core prognostic genes.

546

547 Figure 8. Regulation of core prognosis genes by ncRNA/TF, genes-TF and Target genes-miRNA regulatory 548 network. A. Interaction between core prognostic genes and ncRNA/TF. B. Target genes-TF regulatory network 549 of the 22 hub-gene. Green for TF and red for Hubgene. C. Target genes-miRNA regulatory network of the 22 550 hub-gene. Yellow represents mirna and purple represents Hubgene.

551

552 Figure 9. Mutation analysis of hub genes.

553

554 Figure 10. The ROC curve based on hub genes. A. ENO2, B. GRP, C. HIST1H2AC, D. HIST1H2AK, E. 555 HIST1H4H, F. HIST4H4, G. HOXB4, H. HSPB1, I. MIFT, J. POMC, K. QRFP, L. TNFAIP6. 556

557 Figure 11. Hub gene is associated with immune infiltrating cells. * means $0.01<=\mathrm{P}<0.05$, ** means $\mathrm{P}<0.01$. 558 


\section{Table $\mathbf{1}$ (on next page)}

Table 1 Clinical Information of patients with colorectal cancer from TCGA 
1 Table 1 Clinical Information of patients with colorectal cancer from TCGA

\begin{tabular}{lll}
\hline Parameter & subtype & patients \\
\hline Age & $>66$ & 175 \\
& $<=66$ & 187 \\
Gender & Female & 160 \\
& Male & 202 \\
Stage & I & 55 \\
& II & 130 \\
& III & 110 \\
& IV & 50 \\
OS time(days) & Unknow & 17 \\
& $>700$ & 181 \\
& $<=700$ & 181
\end{tabular}

2 Abbreviations: OS: overall survival; TCGA: The Cancer Genome Atlas 
Table 2 (on next page)

Table 2 Clinical Information of patients with colorectal cancer from GEO 
1 Table 2 Clinical Information of patients with colorectal cancer from GEO

\begin{tabular}{lll}
\hline Parameter & subtype & patients \\
\hline Age & $>70.1$ & 75 \\
& $<=70.1$ & 76 \\
& NA & 1 \\
Gender & Female & 68 \\
& Male & 84 \\
Stage & II & 80 \\
& III & 72 \\
OS time (months) & $>51.2385$ & 76 \\
& $<=51.2385$ & 76 \\
\hline
\end{tabular}

2 Abbreviations: OS: overall survival; GEO: Gene expression omnibus 
Table 3 (on next page)

Table 3 Pivot (ncRNA) 
1 Table 3 Pivot (ncRNA)

\begin{tabular}{lll}
\hline ncRNA & connection & $P$ value \\
\hline MALAT1 & 7 & 0.000346134 \\
CANDE & 6 & 0.000878654 \\
FENDRR & 8 & 0.000451542 \\
TUG1 & 7 & 0.000286648 \\
\hline
\end{tabular}

2 Abbreviations: MALAT1: Metastasis-associated lung adenocarcinoma transcript 1; CRNDE:

3 colorectal neoplasia differentially Expressed; FENDRR: FOXF1 adjacent non-coding

4 developmental regulatory RNA; TUG1: taurine upregulated gene1 
Table 4 (on next page)

Table 4 Pivot (TF) 
1 Table 4 Pivot (TF)

\begin{tabular}{lll}
\hline TF & connection & P value \\
\hline ATF1 & 3 & 0.000124081 \\
CEBPB & 4 & 0.000733944 \\
E2F3 & 2 & 0.00026685 \\
ETV4 & 2 & 0.001742111 \\
PAX3 & 3 & $6.68 \mathrm{E}-07$ \\
SOX10 & 3 & $1.63 \mathrm{E}-05$ \\
SP11 & 2 & 0.035007145 \\
DSF1 & 2 & 0.40192337 \\
USF2 & 2 & 0.18026951 \\
YBX1 & 2 & 0.004397422 \\
YY1 & 3 & 0.021654705
\end{tabular}

2 Abbreviations: ATF1: activating transcription activator 1; CEBPB: CCAAT/enhancer binding proteins C/EBP beta;

3 E2F3: E2F transcription factor 3; ETV4: ETS translocation variant 4; YBX1: Y-box protein 1; PAX3: Paired box 3;

4 SOX10: SRY-related HMG-box 10; SP11: Synaptophysin 11; DSF1: Double-skin façade 1; USF2: Upstream

5 stimulus factor 2; YY1: Yin Yang 1

6 
Table 5 (on next page)

Table 5 Pivot (Drug) 
1 Table 5 Pivot (Drug)

\begin{tabular}{lll}
\hline Drug & connection & P value \\
\hline Pseudoephedrine & 2 & $1.79 \mathrm{E}-05$ \\
Quercetin & 2 & $9.57 \mathrm{E}-05$ \\
\hline
\end{tabular}

2

3 


\section{Figure 1}

Figure 1. The score distribution and the Kaplan-Meier survival curves and the correlation of immune score and stromal score and the stage of CRC

A: the distribution of immune score and stromal score. B: Kaplan-Meier survival curve based on stromal score. C: Kaplan-Meier survival curve based on immune score. D. The correlation of stromals core and the stage of CRC. E. The correlation of stromal score and the stage T of CRC. F. The correlation of immune score and the stage of CRC. G. The correlation of immune score and the stage T of CRC. 

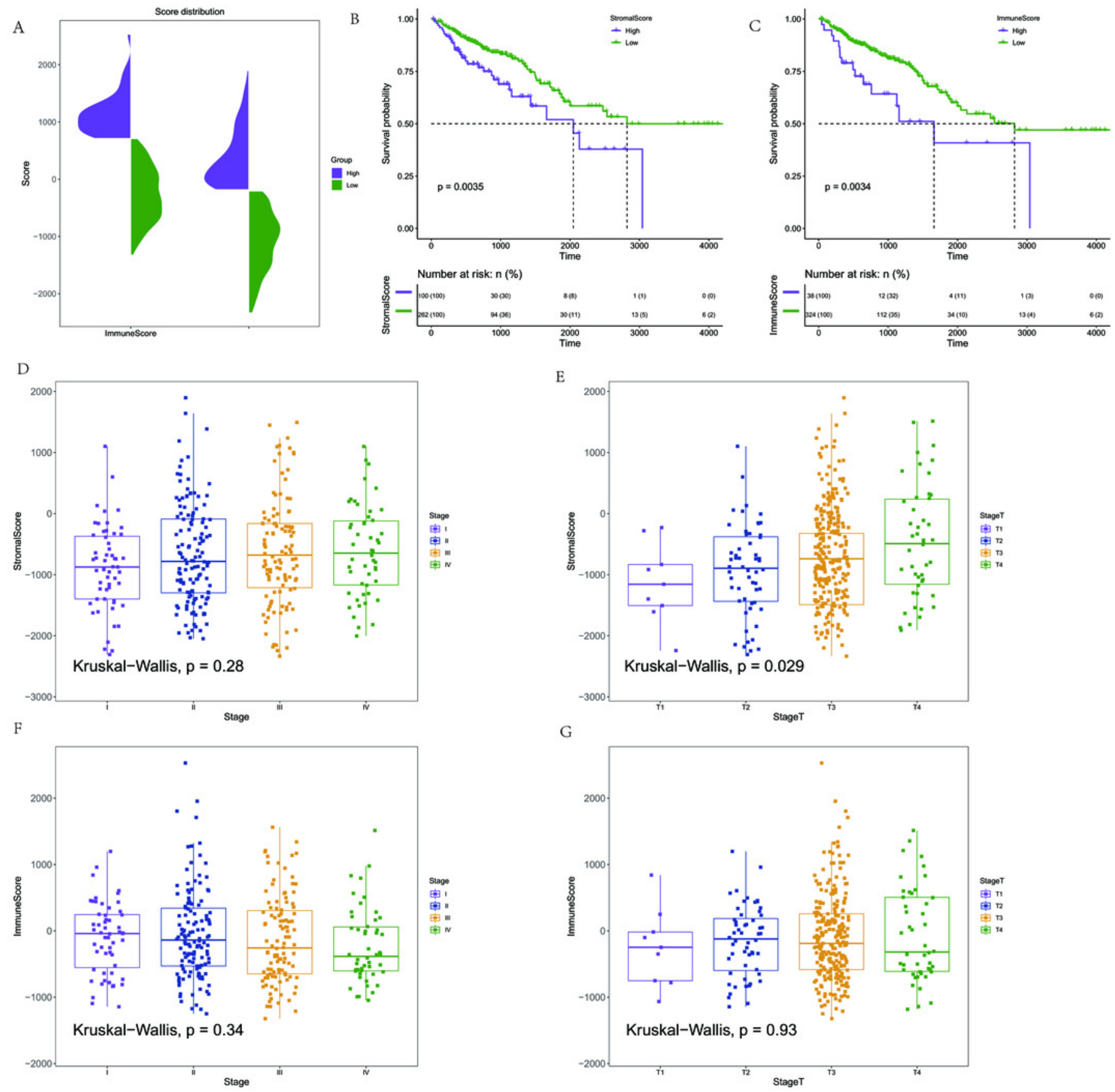


\section{Figure 2}

Figure 2. The heat map and venn diagram of ifferentially expressed genes.

A. Heat map of differential gene expression in gene score. B. Venn diagram. 579 differentially expressed genes were identified according to the immune score groups (38 cases with high score and 324 cases with low score), among which 301 genes were up-regulated and 278 genes were down-regulated. 
A

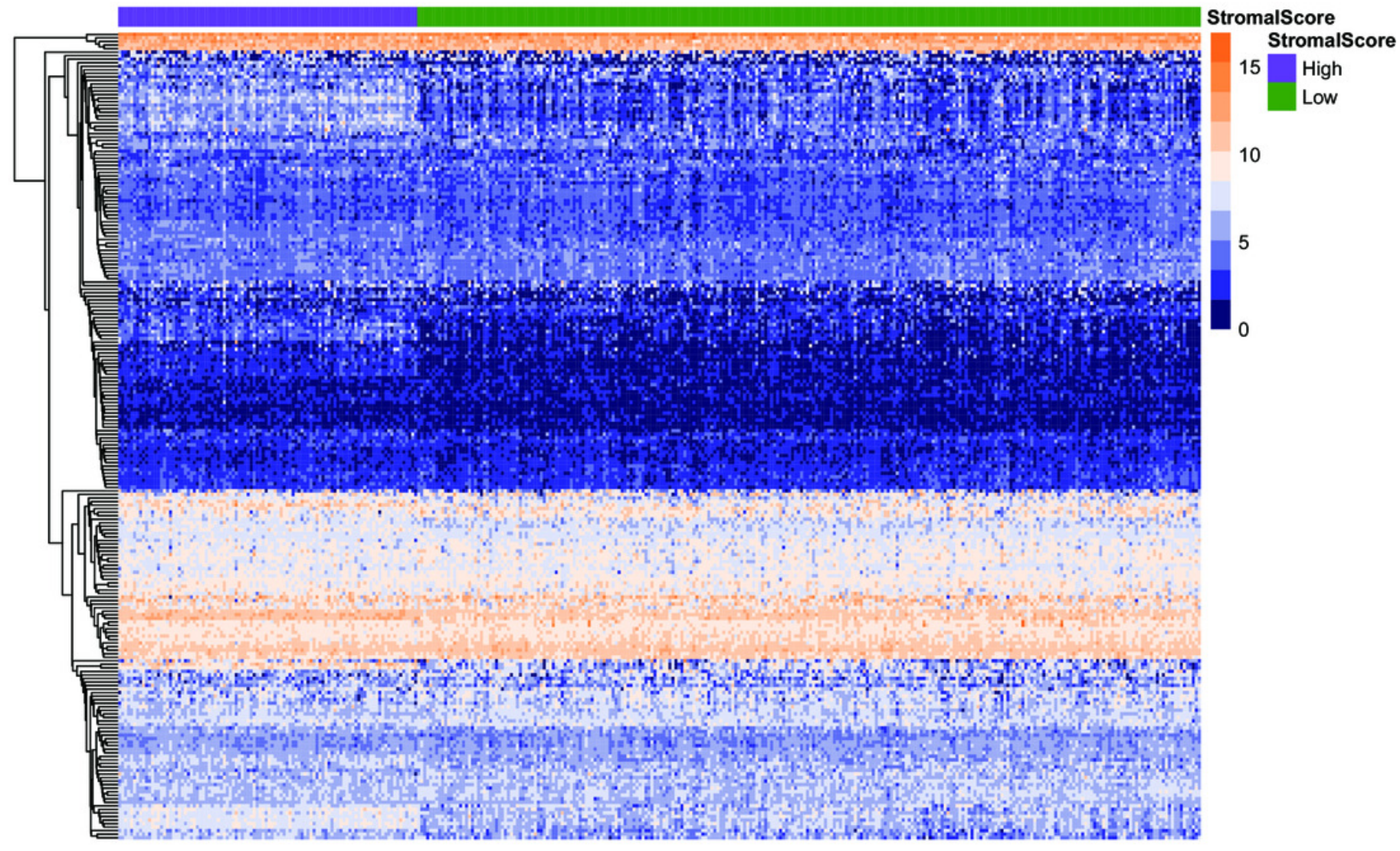

B

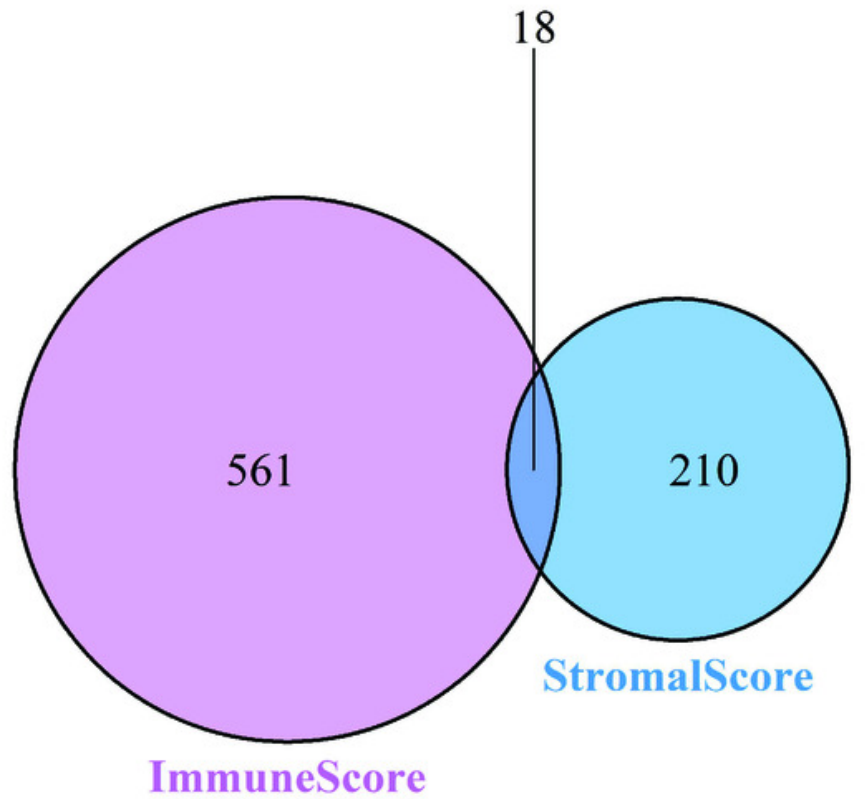


Figure 3

Figure 3. The KEGG and GO analysis of differentially expressed genes.

A. KEGG analysis of the 789 differentially expressed genes (DEGs). B. GO Biological Process of the 789 differentially expressed genes (DEGs).

A

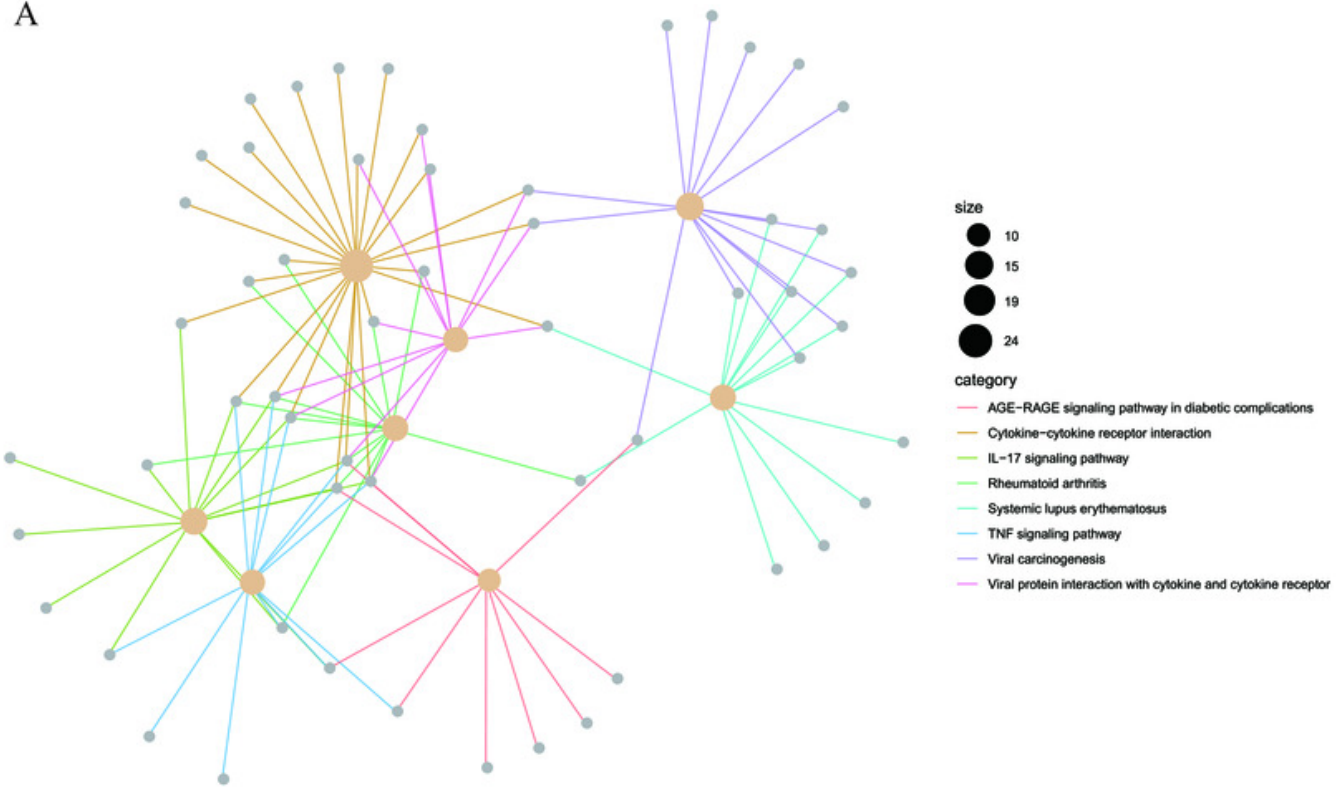

B

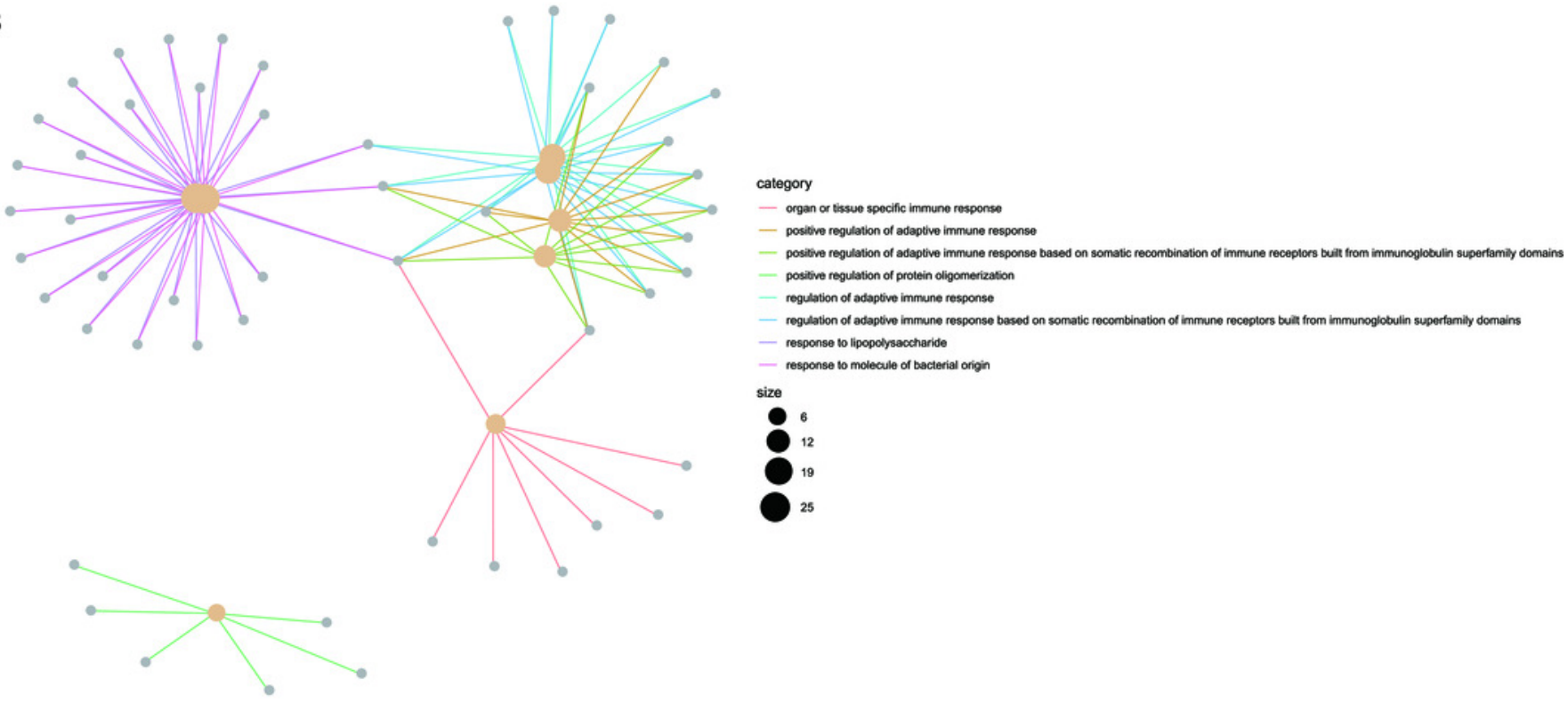


Figure 4

Figure 4. The results of the enrichment analysis of KEGG pathway in GSEA.

A. Enrichment analysis based on immunescore. B. Enrichment analysis based on stromalscore. 

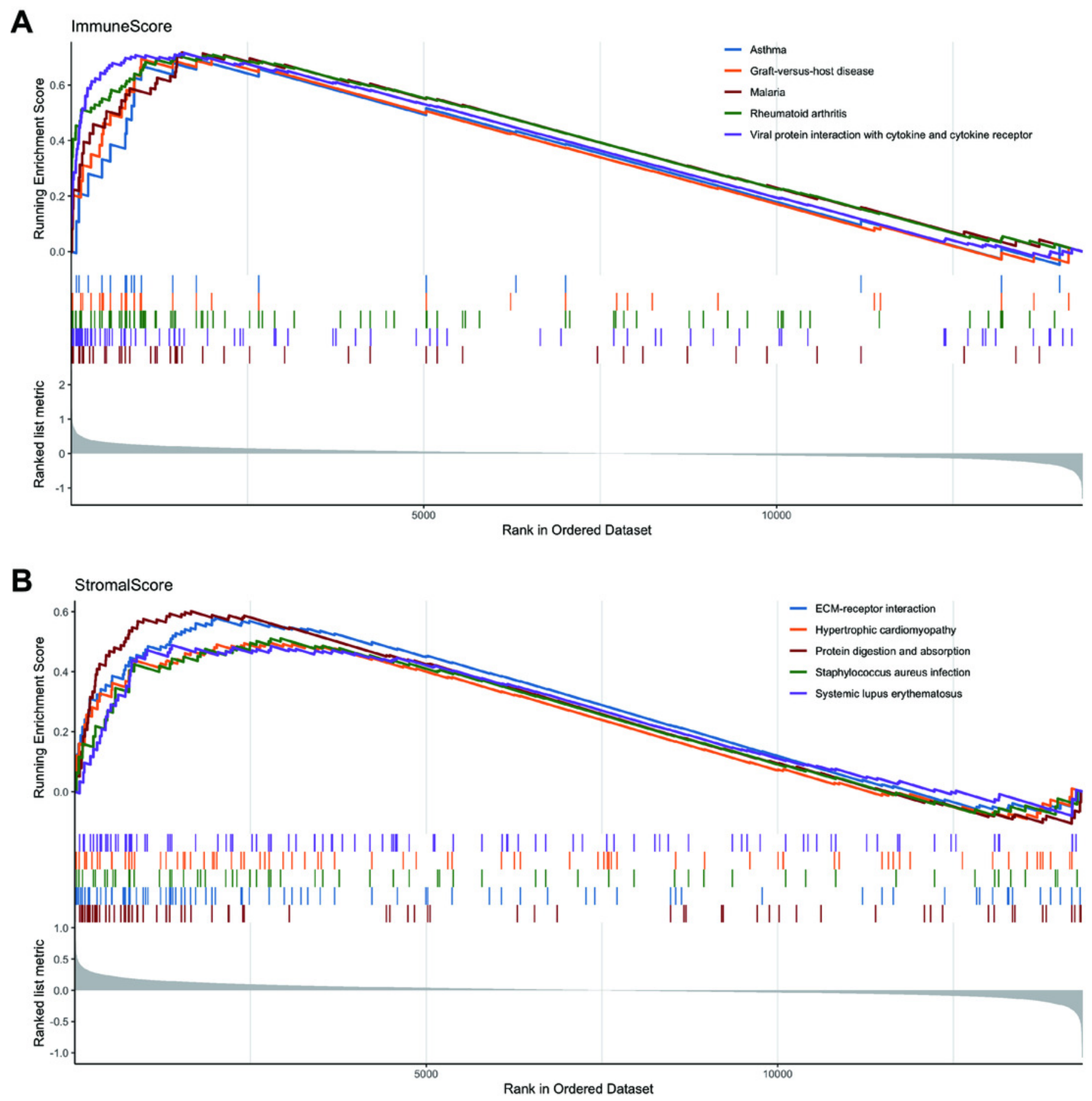


\section{Figure 5}

Figure 5. The Kaplan-Meier survival curve of the PRIM1 gene.

A. Kaplan-Meier survival curve of the PRIM1 gene (TCGA database). A total of 121 survivalrelated prognostic genes were extracted $(p<0.05)$. B. Kaplan-Meier survival curve of the PRIM1 gene (GEO database). 
A

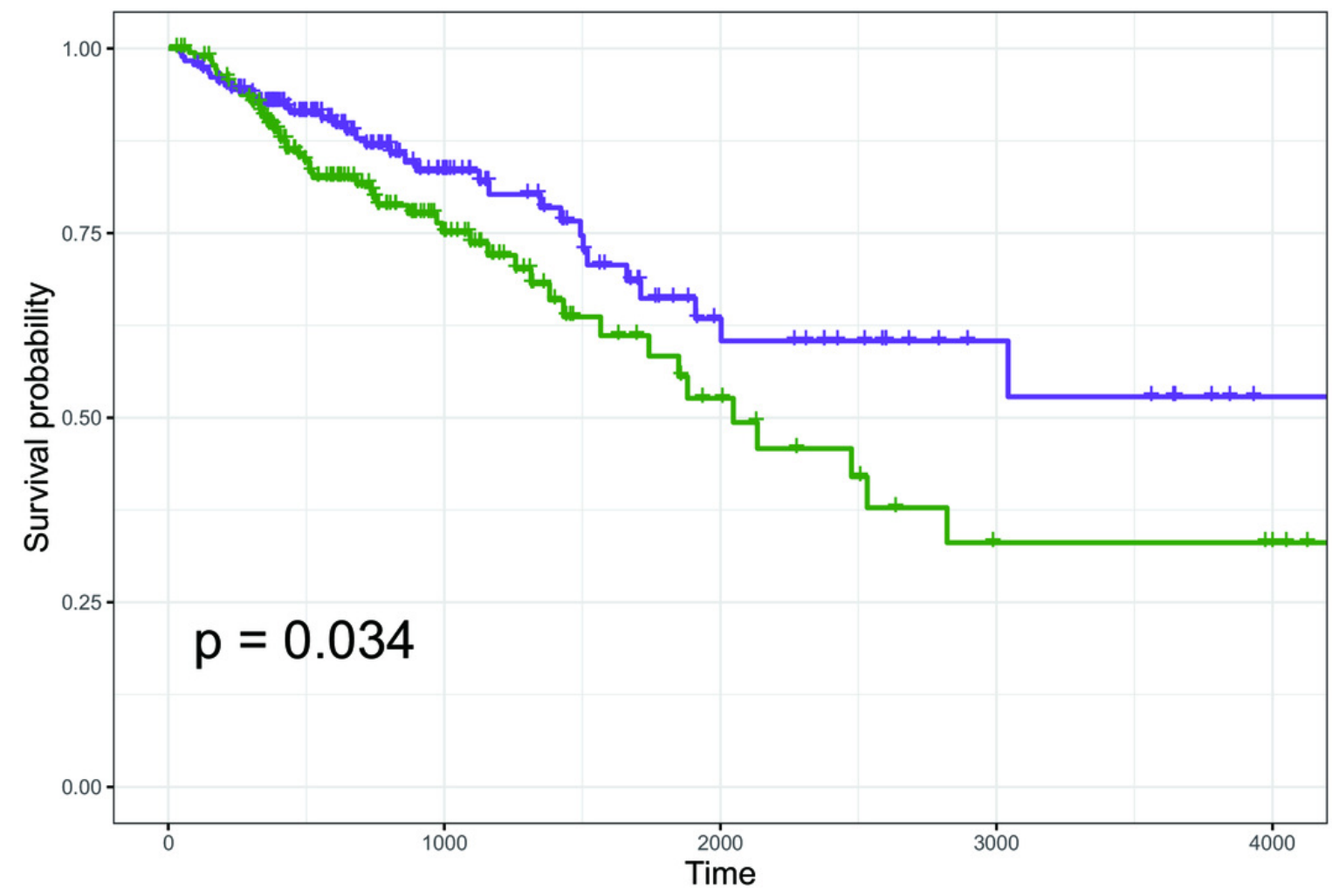

B.
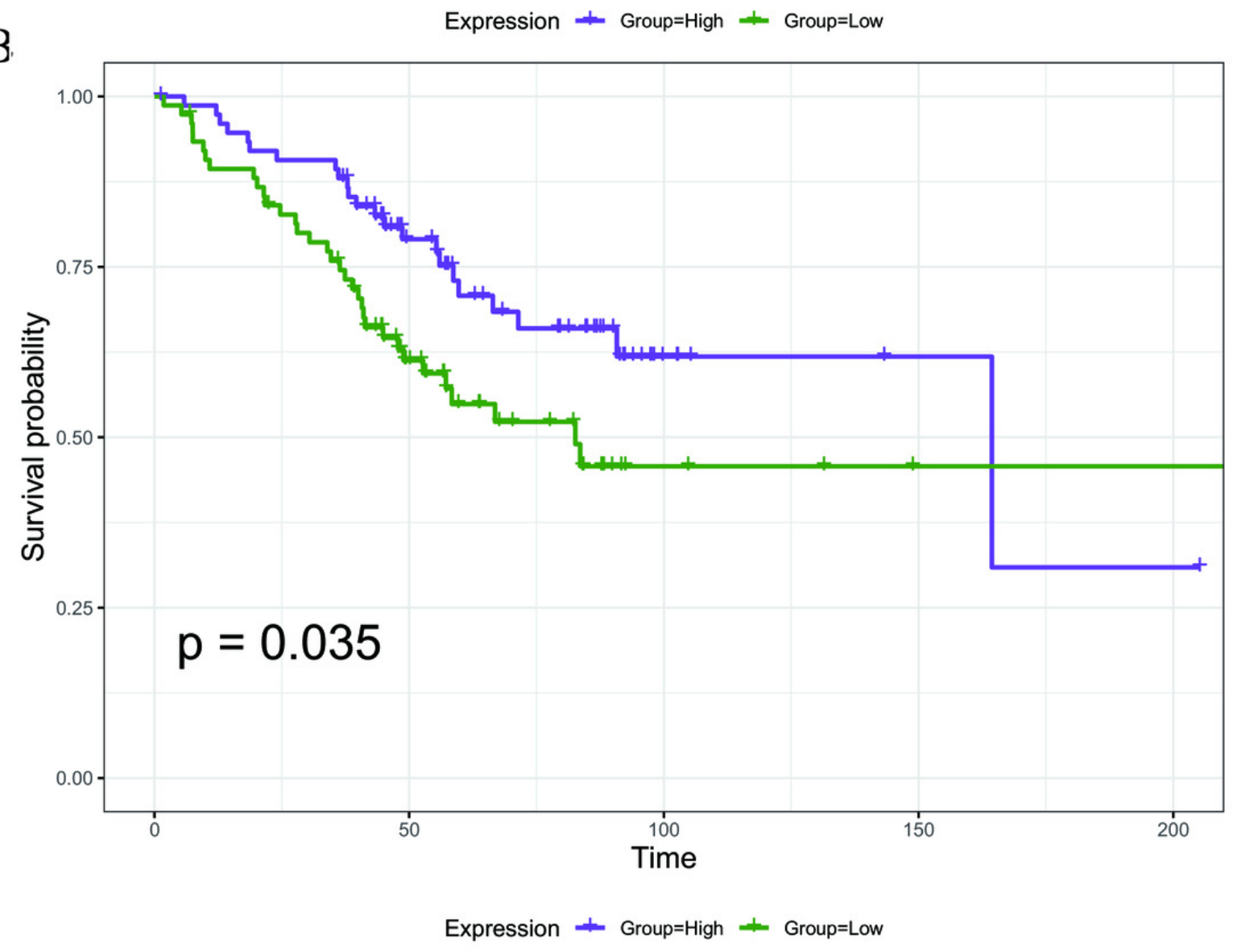
Figure 6

Figure 6. PPI network. The PPI network consisting of 35 nodes and 36 edges.

A total of 22 core genes that associated with CRC prognosis were defined.
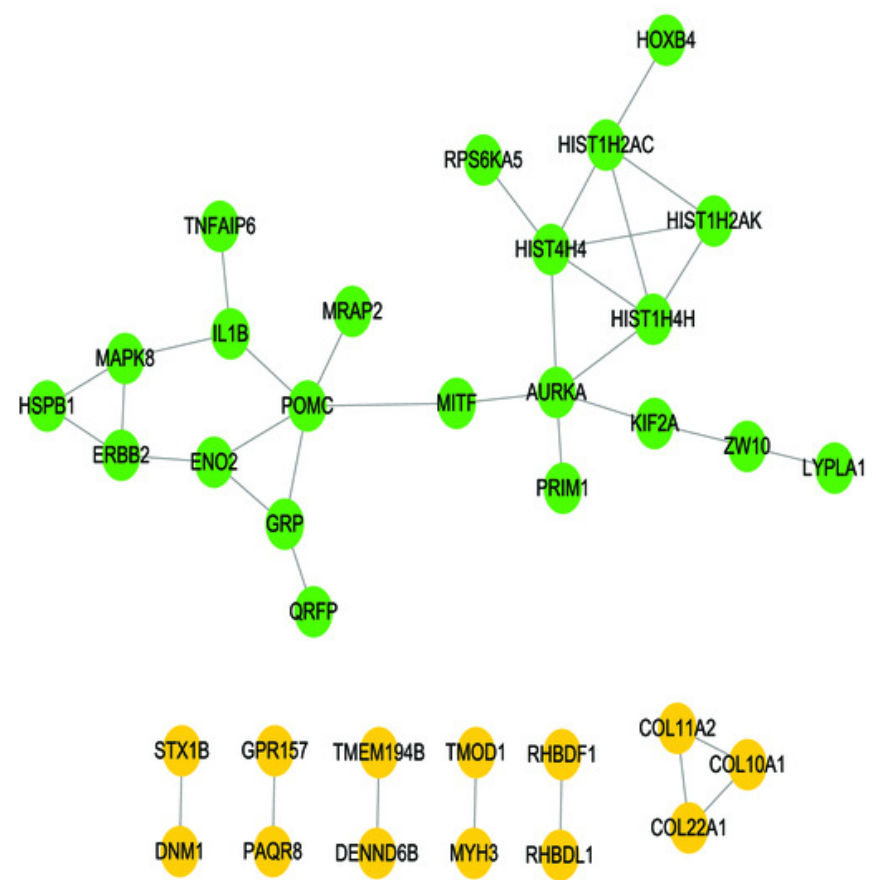
Figure 7

Figure 7. The KEGG and GO analysis of 22 core prognostic genes.

A. KEGG analysis of the 22 core prognostic genes. B. GO Biological Process of the 22 core prognostic genes.

A

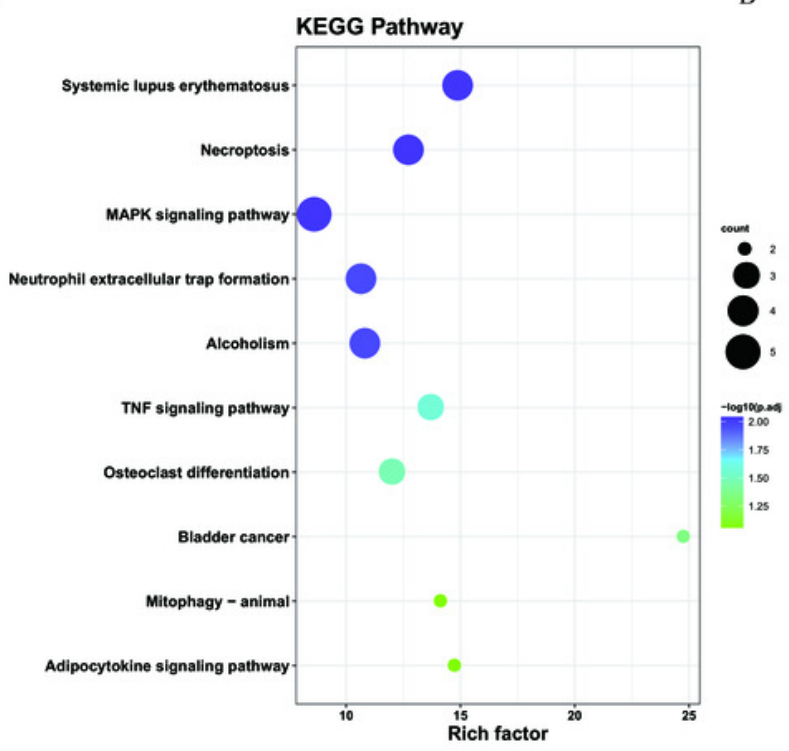

B

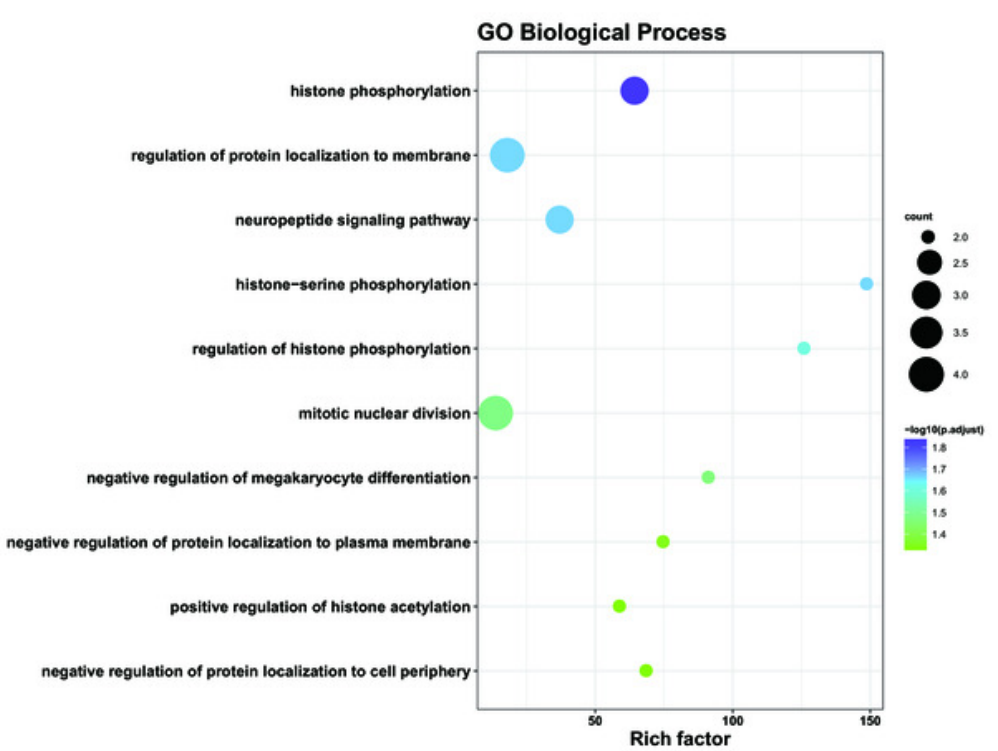




\section{Figure 8}

Figure 8. Regulation of core prognosis genes by ncRNA/TF, genes-TF and Target genesmiRNA regulatory network.

A. Interaction between core prognostic genes and ncRNA/TF. B. Target genes-TF regulatory network of the 22 hub-gene. Green for TF and red for Hubgene. C. Target genes-miRNA regulatory network of the 22 hub-gene. Yellow represents mirna and purple represents Hubgene. 
A

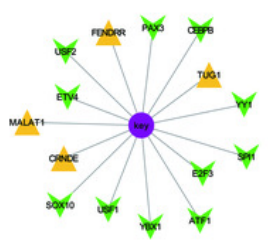

B

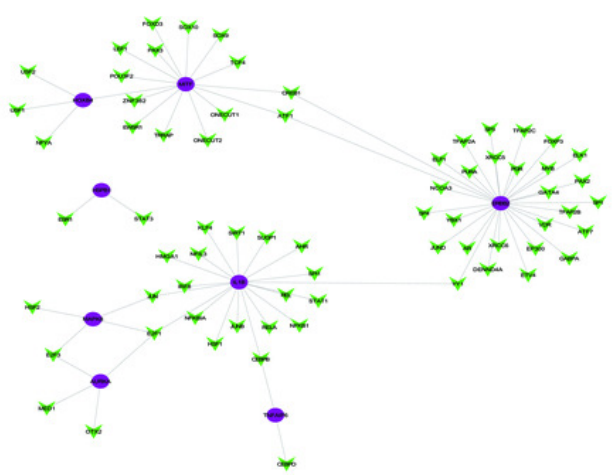

C

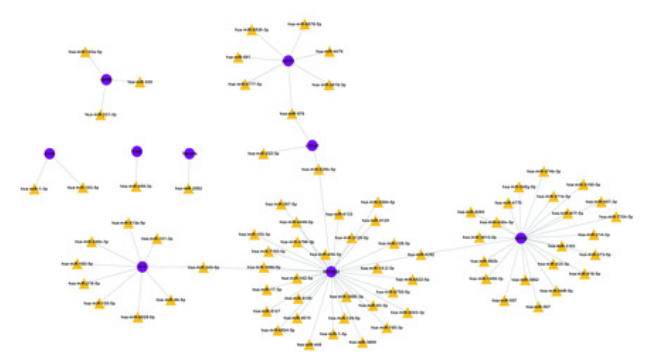


Figure 9

Figure 9. Mutation analysis of hub genes

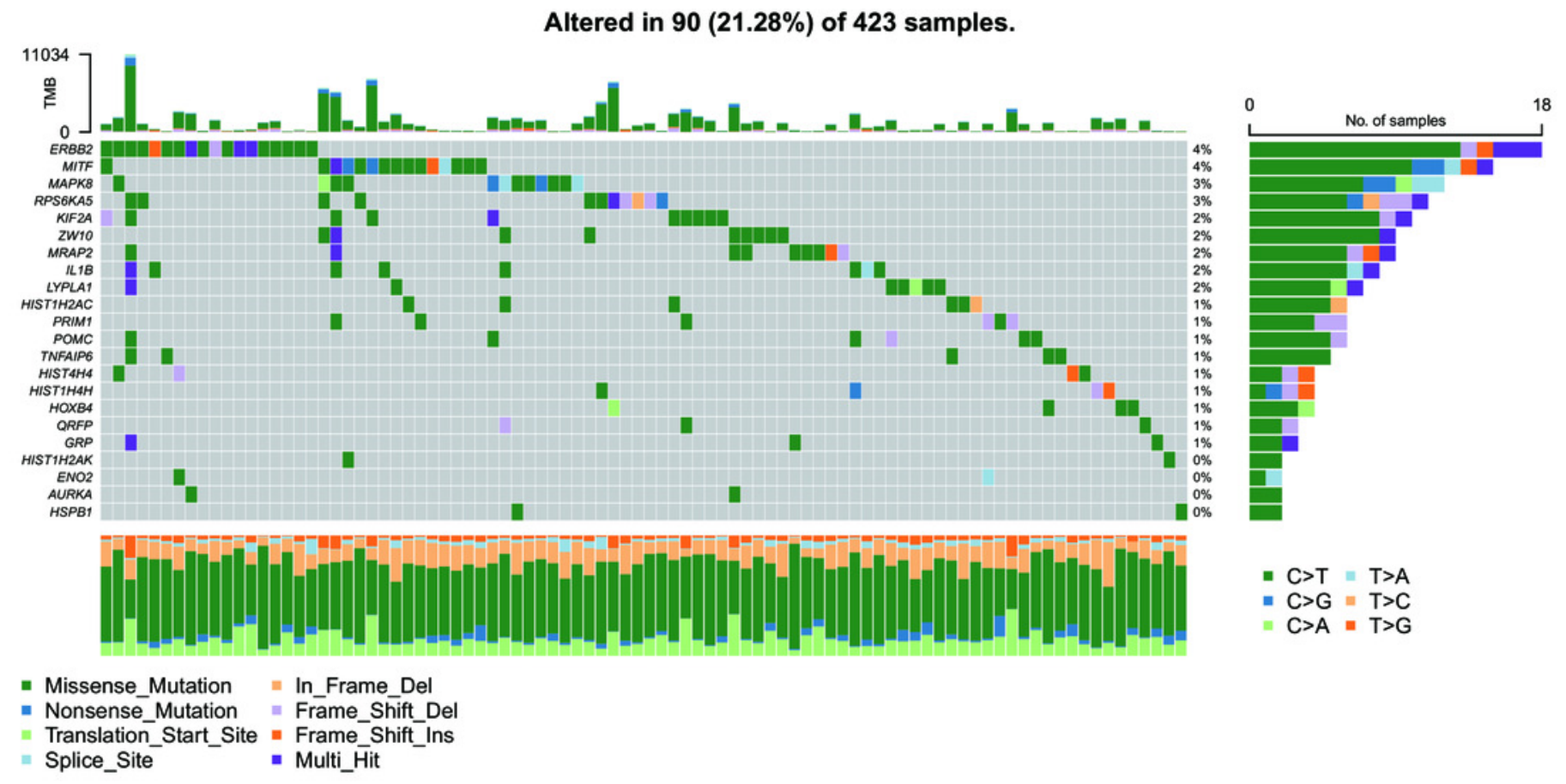


Figure 10

Figure 10. The ROC curve based on hub genes.

A. ENO2, B. GRP, C. HIST1H2AC, D. HIST1H2AK, E. HIST1H4H, F. HIST4H4, G. HOXB4, H. HSPB1, I. MIFT, J. POMC, K. QRFP, L. TNFAIP6.
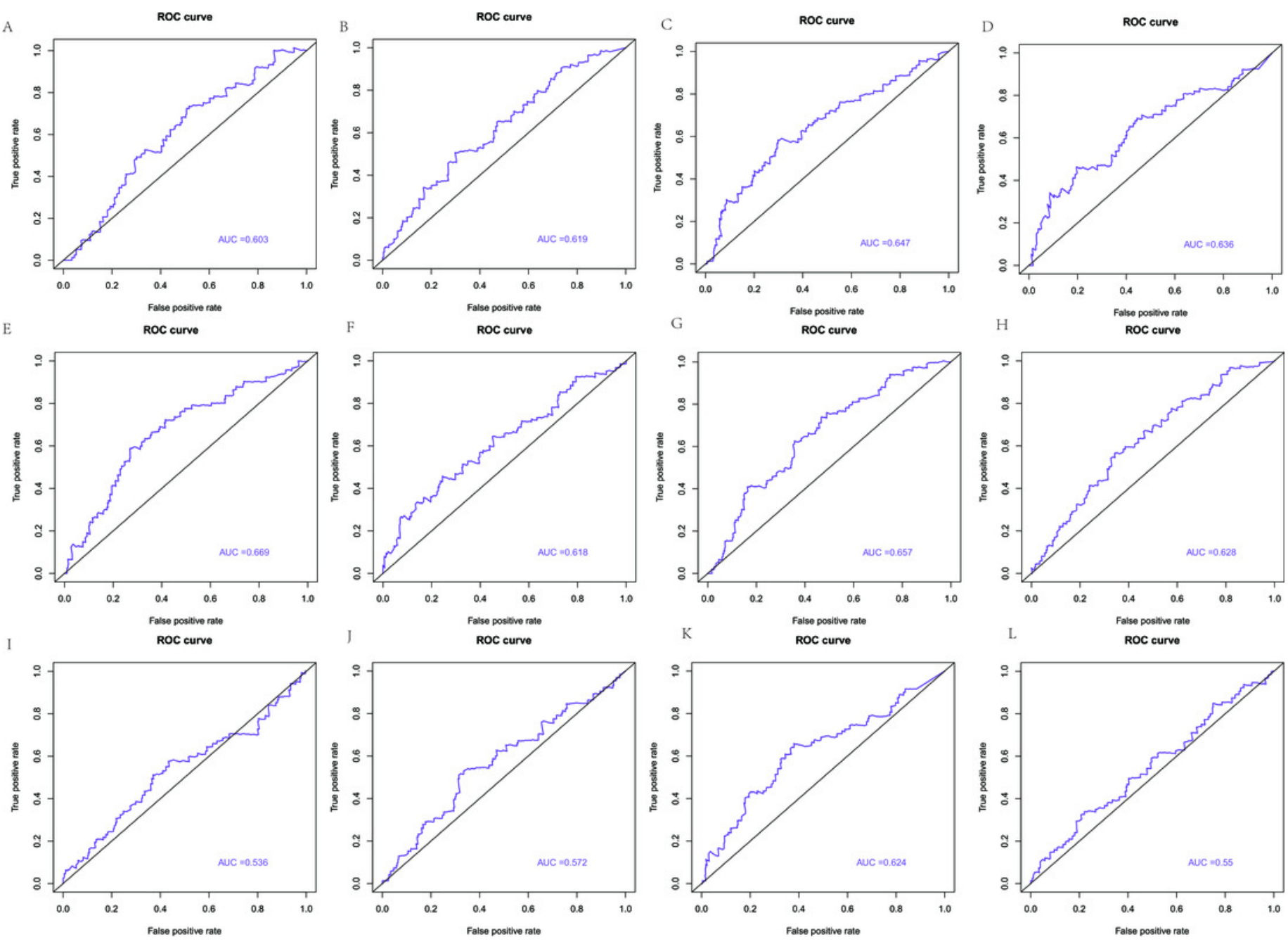
Figure 11

Figure 11. Hub gene is associated with immune infiltrating cells.

* means $0.01<=\mathrm{P}<0.05$, ** means $\mathrm{P}<0.01$.

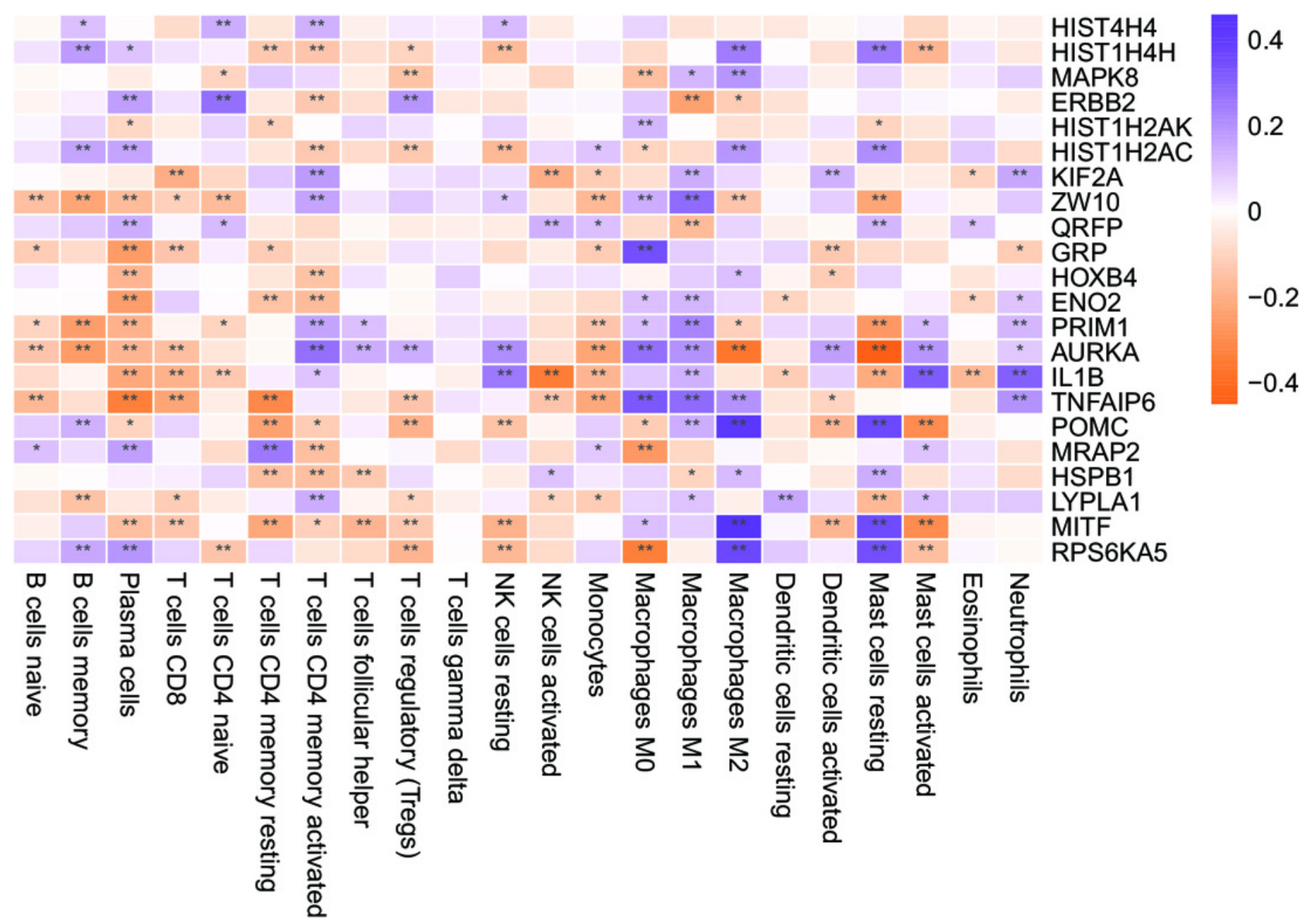

